Trauma Berufskrankh 2008 · 10 [Suppl 3]:357-365

DOI 10.1007/s10039-008-1365-1

Online publiziert: 7. März 2008

(c) Springer Medizin Verlag 2008

\author{
M. Kusma · F. Bachelier · D. Kohn · M. Dienst \\ Klinik für Orthopädie und Orthopädische Chirurgie, \\ Universitätskliniken des Saarlandes, Homburg, Saar
}

\title{
Operative Arthroskopie des Hüftgelenks
}

\section{Technik und Indikationen}

Das Interesse an der Arthroskopie des Hüftgelenks ist in den letzten Jahren sprunghaft angestiegen. Hierfür sind neben dem allgemeinen Trend zur minimalinvasiven Therapie v. a. die technische Weiterentwicklung der Instrumente sowie neue pathogenetische Konzepte wie das femoroazetabuläre Impingement (FAI) verantwortlich.

Die zunehmende Erfahrung mit der arthroskopischen und offenen Gelenk erhaltenden Hüftchirurgie hat zu einem besseren Verständnis der Funktion und Anatomie des normalen Hüftgelenks, seiner Normvarianten und krankhaften Veränderungen und damit zur Spezifizierung der Indikationen zur arthroskopischen oder offenen Gelenk erhaltenden Hüftchirurgie geführt. Im vorliegenden Beitrag werden wichtige Grundlagen der Hüftarthroskopie beschrieben, arthroskopische und offene Therapieverfahren voneinander abgegrenzt und sinnvolle Indikationen für ein arthroskopisches Vorgehen vorgestellt (『 Tab. 1).

\section{Anatomische Grundlagen}

Der arthroskopische Zugang und die Beweglichkeit von Arthroskop und Instrumenten im Hüftgelenk sind im Vergleich zu anderen Gelenken erschwert. Dafür sind verschiedene anatomische Eigenheiten des Hüftgelenks verantwortlich:

- der dicke Weichteilmantel,

- die Nähe zu 2 neurovaskulären Bündeln,

- eine kräftige Gelenkkapsel,
- ein vergleichsweise kleiner Binnenraum,

- der permanente Kontakt von Femurkopf und Pfanne und

- die zusätzliche Abdichtung des "tiefen" Gelenkanteils durch das Labrum acetabulare.

Ohne Zug am Bein sind die Gelenkflächen von Azetabulum und Femur nur durch einen dünnen Flüssigkeitsfilm getrennt.

Bei der Anlage der Portale zur Arthroskopie des Hüftgelenks muss insbesondere die Anatomie des Labrum acetabulare berücksichtigt werden. Es dich- tet den „tiefen“ Gelenkanteil ab, sodass sogar unter vollständiger Muskelrelaxation eine Vakuumkraft zwischen 120 und $200 \mathrm{~N}$ aufrechterhalten wird [21, 54]. Zur Separation von Femurkopf und Gelenkpfanne und damit Überwindung des Vakuums und Dehnung der Gelenk überbrückenden Weichteile bedarf es der Anlage von nicht unerheblichen Zugkräften, die nicht selten über 400-500 N liegen. Nur so lässt sich das Labrum weit genug vom Femurkopf wegziehen, um Arthroskop und Instrumente zwischen Kopf und Pfanne einzubringen [10]. Auf der anderen Seite führt die Traktion zur Anspan-

Tab. 1 Sinnvolle Indikationen zur arthroskopischen und offenen Hüftchirurgie

\begin{tabular}{|c|c|c|}
\hline Indikationen & Arthroskopie & Offene/chirurgische Luxation \\
\hline Unklare Hüftschmerzen & +++ & $\oslash$ \\
\hline Freie Körper & +++ & Ggf. bei sehr großen Körpern \\
\hline Labrum & $\begin{array}{l}\text { Teilresektion/Resektion } \\
\text { Ggf. Refixation (1-2 Anker) }\end{array}$ & $\begin{array}{l}\text { Refixation über größere Strecke bei } \\
\text { temporärer Ablösung (Pincer-FAl) }\end{array}$ \\
\hline Gelenkknorpel & $\begin{array}{l}\text { Abrasion/Mikrofrakturierung } \\
\text { Diagnostik/Gelenkstatus }\end{array}$ & $\begin{array}{l}\text { Ggf. für Mosaikplastik } \\
\text { Ggf. für ACT }\end{array}$ \\
\hline Lig. capitis femoris & +++ & $\oslash$ \\
\hline Synoviale Erkrankungen & $\begin{array}{l}\text { Synovitis } \\
\text { Osteo-/Chondromatose } \\
\text { Lokale, ggf. diffuse PVNS }\end{array}$ & $\begin{array}{l}\text { Osteochondromkonglomerate } \\
\text { Ggf. Chondromatoserezidiv } \\
\text { Ggf. diffuse PVNS, PVNS-Rezidiv }\end{array}$ \\
\hline FAl & $\begin{array}{l}\text { Cam ventral/ventrolateral } \\
\text { Initialer Pincer } \\
\text { Pincer + Labrumverkalkung }\end{array}$ & $\begin{array}{l}\text { Cam nach lateral } \\
\text { Pincer } \\
\text { Deformität proximales Femur }\end{array}$ \\
\hline Psoastenotomie & +++ & $\oslash$ \\
\hline Infektiöse Arthritis & ++, Etappen-ASK & $?$ \\
\hline Hüftkopfnekrose & $?-\oslash$ & Nach Stadium \\
\hline Morbus Perthes & $\begin{array}{l}\text { Freie Körper } \\
\text { Hinge abduction }\end{array}$ & Umstellungsosteotomie \\
\hline TEP-Komplikation & Psoastenotomie & Andere Komplikationen \\
\hline
\end{tabular}



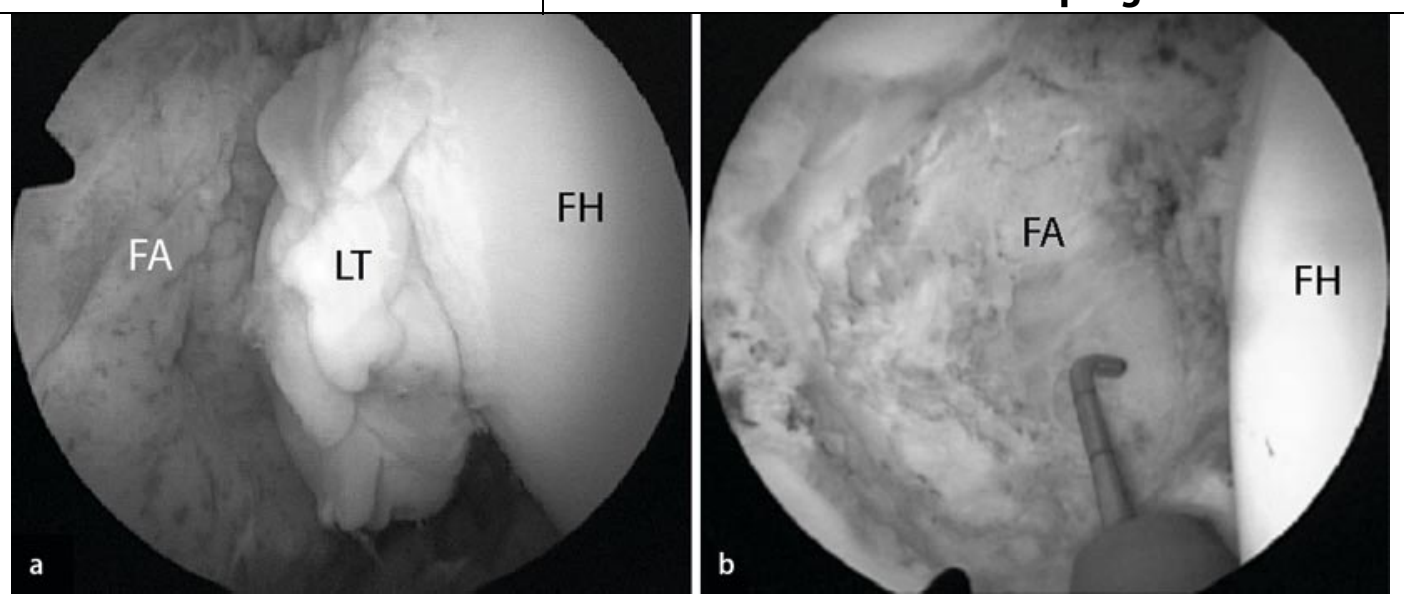

Abb. $1<$ Nichttrauma-

tische Ruptur des Lig. capitis femoris bei Hüftdysplasie vor (a) und nach Resektion (b), FA Fossa acetabuli, FH Femurkopf, $L T$ Lig. capitis femoris. (Mit Genehmigung von M. Dienst)

nung der Gelenkkapsel und ihrer intrinsischen Ligamente, dem Lig. iliofemorale, Lig. ischiofemorale und Lig. pubofemorale, sodass der Gelenkraum peripher zum Labrum acetabulare abnimmt. Zudem kann das Hüftgelenk unter Traktion mit Ausnahme der Rotation kaum bewegt werden. Die Arthroskopie des peripheren Gelenkbereichs ohne Traktion mit beweglich abgedecktem Hüftgelenk bietet damit große Vorteile [19].

Die beschriebenen anatomischen $\mathrm{Ge}$ gebenheiten führten zur Unterteilung des Hüftgelenks durch Dorfmann u. Boyer [24] in 2 arthroskopische Kompartimente. Das zentrale Kompartiment umfasst:

- die azetabuläre Gelenkfläche,

- die Fossa acetabuli mit Pulvinar und Lig. capitis femoris und

- die vorwiegend belastete Gelenkfläche des Femurkopfs.

Hiervon abgetrennt durch das Labrum acetabulare finden sich im peripheren Kompartiment:

- die meist nicht belastete Knorpelfläche des Femurkopfs,

- der intraartikulär liegende Schenkelhals mit seinen Synovialfalten,

- ein Großteil der Gelenkschleimhaut und

- die Gelenkkapsel mit ihren intrinsischen Ligamenten und der Zona orbicularis.

\section{Technische Grundlagen}

\section{Lagerung}

Die Hüftarthroskopie kann sowohl in Rücken- [5] als auch in Seitenlage [28] durchgeführt werden. Eigene Studien und Hos- pitationen ergaben, dass die Lagerungsart keinen signifikanten Einfluss auf die Distraktion des Hüftgelenks hat [53], unserer Erfahrung nach bietet die Rückenlage Vorteile bei der Arthroskopie ohne Traktion. Es ist allerdings festzuhalten, dass der jeweiligen Lagerungstechnik mit dicker Polsterung unter der Fußmanschette, korrekter Positionierung des Gegenzugstabs und Ausrichtung der Traktionsausleger eine wesentlich umfassendere Bedeutung zukommt, insbesondere auch um Lagerungskomplikationen zu vermeiden. Der Einsteiger in die arthroskopische Hüftchirurgie sollte bei der Wahl der Lagerungsart berücksichtigen, welche Orientierung er bei der offenen Gelenk erhaltenden und ersetzenden Hüftchirurgie gewohnt ist.

Die kombinierte Technik der Arthroskopie des Hüftgelenks mit und ohne Traktion mit beweglichem Hüftgelenk erfordert eine besondere Lagerungs- und Vorgehensweise, zu deren Technik auf die weiterführende Literatur verwiesen wird $[15,20]$.

\section{Zugang}

Zur korrekten Portalanlage muss ein Röntgenbildverstärker benutzt werden, zumindest um ein Erstportal zu etablieren. Bei entsprechender Erfahrung ist es möglich, alle weiteren Portal unter arthroskopischer Kontrolle anzulegen [22].

Zur Inspektion und Behandlung pathologischer Veränderungen des Hüftgelenks müssen für jedes Kompartiment in der Regel jeweils 3 Portale etabliert werden $[13,15]$. Das zentrale Kompartiment kann nahezu vollständig über ein ventrales, ventrolaterales und dorsolaterales
Portal eingesehen und instrumentiert werden. Häufig finden sich hier Schäden des ventrolateralen Labrum-Knorpel-Übergangs, welche mit Shaver und Instrumenten nur über die Kombination des ventralen und ventrolateralen Portals zu behandeln sind. In der Fossa acetabuli sind nicht selten pathologische Veränderungen wie freie Körper, Synovitiden und Rupturen des Lig. capitis femoris, die vollständig nur über das ventrale und dorsolaterale Portal zu erreichen sind. In dysplastischen und sehr laxen Gelenken kann die Anlage von 2 Portalen ausreichend sein. Für das periphere Kompartiment verwenden wir ein ventrales und ein proximales und distales ventrolaterales Portal. Insbesondere zur Behandlung des femoroazetabulären Impingements sind 3 Portale vorteilhaft, um eine korrekte Resektion des Kopf-Hals-Übergangs unter Schonung der Kopf versorgenden Blutgefäße zu kontrollieren.

\section{Inspektion}

Wie in allen anderen Gelenken sollte auch im Hüftgelenk das Durchscannen der verschiedenen Gelenkbereiche standardisiert werden [17]. Nur so können pathologische Veränderungen sicher ausgeschlossen, diagnostiziert und von Normvarianten abgegrenzt werden. In beiden Gelenkkompartimenten wird dazu das Arthroskop über jedes Portal eingeführt, hierbei ist je nach Sichtbedingungen und Kompartiment auch der Wechsel zwischen der $30^{\circ}$ und $70^{\circ}$-Optik notwendig. Insbesondere im zentralen Kompartiment wird die $70^{\circ}$ Optik zur Hauptoptik, v. a. um das Labrum und den Übergang zwischen Labrum und Facies lunata zu inspizieren. Für die 
Fossa acetabuli und das periphere Kompartiment verwenden wir vorwiegend die $30^{\circ}$-Optik.

\section{Indikationen}

\section{„Unklare“ Hüftschmerzen}

Seit Standardisierung der präoperativen Röntgendiagnostik, Verbesserung der MR-Tomographen und Einführung der MR-Arthrographie ist diese Indikationsstellung in den Hintergrund getreten. Dennoch ist festzuhalten, dass selbst durch hochauflösende MR-Arthrogramme pathologische Veränderungen wie nichtdislozierte Knorpellappenläsionen und Rupturen des Lig. capitis femoris oft nicht erkannt werden ( $\mathbf{D}$ Abb. 1). Zudem wiesen mehrere Arbeiten darauf hin, dass die Hüftarthroskopie in der Diagnose von intraartikulären Läsionen radiologischen Verfahren überlegen ist [16].

Im Hinblick auf die erhebliche Invasivität eines offenen Vorgehens ist der „unklare“ Hüftschmerz ohne durchgreifende Besserung durch krankengymnastisch-physikalische und antiphlogistische Maßnahmen eine klare Indikation zur Arthroskopie. Bei der chirurgischen Luxation sind subtile Veränderungen nur unzureichend $\mathrm{zu}$ erfassen, zudem muss hier$\mathrm{zu}$ u. a. das Lig. capitis femoris geopfert werden. Über die Diagnosestellung hinaus kann durch die Arthroskopie die pathologische Veränderung direkt behandelt werden. Insbesondere bei unklaren Hüftbeschwerden ist es obligat, beide Kompartimente des Hüftgelenks mit und ohne Traktion vollständig zu arthroskopieren.

\section{Freie Körper}

Ihr radiologischer Nachweis, mit entsprechenden klinischen Symptomen, stellt eine klassische Indikation für ein arthroskopisches Verfahren an der Hüfte dar [28, 29, 30, 36, 49, 50, 51]. Freie Körper finden sich nicht selten:

- nach einem Anpralltrauma [7],

- auf Basis einer Koxarthrose [18],

- bei synovialer Chondromatose [41, 43, 55],

- beim Morbus Perthes [33, 37] und

- bei einer Osteochondrosis dissecans [31].
Arthroskopisch können meist auch größere Körper entfernt werden, die vorher mit geeigneten Instrumenten zerkleinert werden müssen ( $\bullet$ Abb. 2). Insbesondere die Entfernung größerer knöcherner Osteochondrome in der Fossa acetabuli kann technisch sehr anspruchsvoll sein, sie können nur über die Anlage einer Kanüle mit einem größeren Innendurchmesser bis vor die Gelenkkapsel und Einbringen eines kräftigeren Instruments wie einem Rangeur geborgen werden. Je nach Lagerungsart sammeln sich freie Körper bevorzugt in den dorsalen oder medialen Gelenkanteilen, oft versteckt unter den Schleimhautfalten im peripheren Kompartiment an. Je nach Anzahl, Lage oder Größe der freien Körper ist alternativ ein offenes oder arthroskopisch-assistiertes Vorgehen zu erwägen. Die Ergebnisse der arthroskopischen Entfernung von freien Körpern sind meist sehr gut [28, 29, 30, $36,49,50,51]$.

\section{Labrumläsionen}

Die isolierte Labrumruptur durch ein niedrigenergetisches Trauma ist eine $\mathrm{Ra}$ rität. Das Risiko einer isolierten Labrumund Knorpelverletzung nach einer Hüftluxation ist dagegen groß. Beim radiologischen Verdacht auf eine Veränderung des Labrums eines Patienten ohne hochenergetisches Trauma ist daher immer auf eine andere pathologische Veränderung des Hüftgelenks zu achten. An erster Stelle stehen hier:

- das femoroazetabuläre Impingement,

- sekundäre Kopfdeformitäten wie nach einer Epiphyseolysis capitis femoris und dem Morbus Perthes und die Hüftdysplasie.

In diesem Zusammenhang sei auch darauf hingewiesen, dass meist der sublabrale Knorpel und nicht direkt das Labrum acetabulare betroffen ist. Ausgedehnte Labrumschäden und instabile Längsrisse finden sich häufiger bei der Hüftdysplasie.

In Assoziation mit einem femoroazetabulären Impingement finden sich die Läsionen des Knorpel- und Labrumübergangs meist ventrosuperior, am stehenden Patienten zwischen 11 und 15 Uhr am rechten Hüftgelenk (- Abb. 3). Bei der Hüftdysplasie reichen sie weiter bis nach
Trauma Berufskrankh 2008 10

[Suppl 3]:357-365

DOI 10.1007/s10039-008-1365-1

○) Springer Medizin Verlag 2008

M. Kusma - F. Bachelier · D. Kohn · M. Dienst

\section{Operative Arthroskopie des Hüftgelenks. Technik und Indikationen}

\section{Zusammenfassung}

Die Arthroskopie des Hüftgelenks hat zu einem besseren Verständnis der funktionellen Anatomie und ihrer pathologischen Veränderungen geführt. Nicht nur der allgemeine Trend zur minimalinvasiven Therapie, auch die Entwicklung neuer Instrumente und pathogenetische Konzepte haben eine rasante Verbesserung der technischen Möglichkeiten mit völlig neuen therapeutischen Optionen nach sich gezogen. Der vorliegende Beitrag gibt einen Überblick über die Grundlagen der arthroskopischen Technik mit anschließendem Fokus auf sinnvolle Indikationen zur Hüftarthroskopie.

\section{Schlüsselwörter}

Hüftgelenk · Arthroskopie · Minimalinvasive Technik · Therapeutische Optionen . Indikationen

\section{Operative hip arthroscopy. Techniques and indications}

\section{Abstract}

Arthroscopy of the hip joint has contributed to better understanding of the functional anatomy and pathologic conditions of this joint. The need for minimally invasive approaches, the development of better instrumentation, and new concepts such as femoroacetabular impingement have led to tremendous improvements of this technique and opened up new therapeutic options. This report presents the basic techniques and indications for hip arthroscopy with and without traction of the central and peripheral compartment of the hip.

\section{Keywords}

Hip · Arthroscopy · Minimally invasive technique $\cdot$ Therapeutic options · Indications 


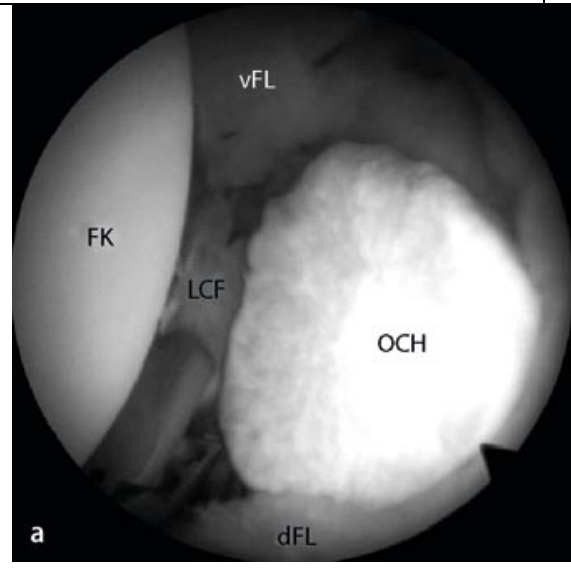

Indikationen zur Arthroskopie großer Gelenke

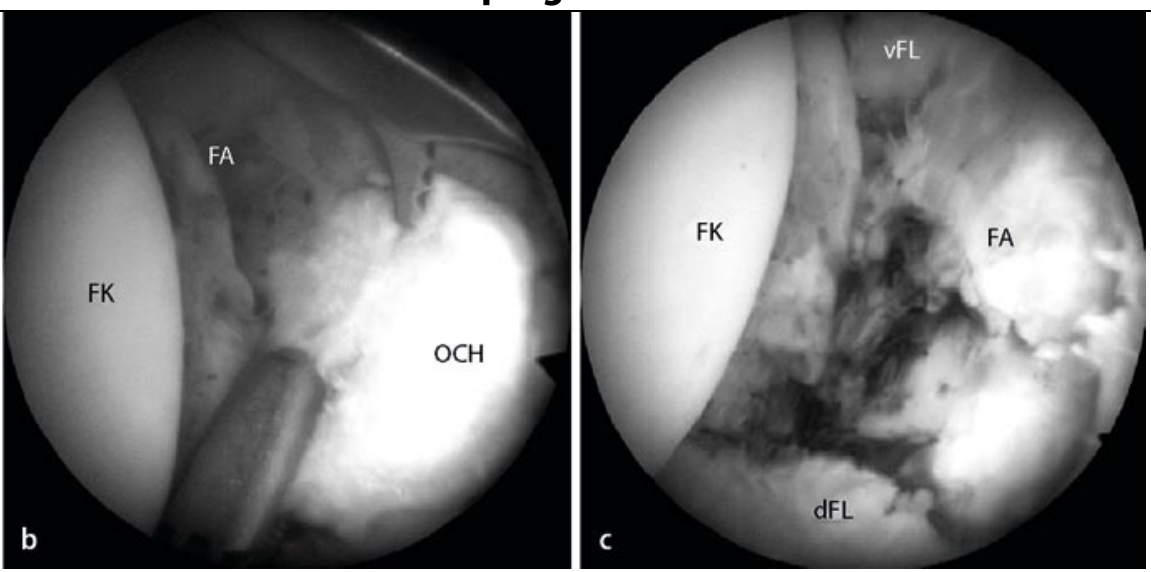

Abb. $2 \Delta$ Großes Osteochondrom, a Lokalisation in der Fossa acetabuli, b Fixieren von ventral und Zerkleinern mit einer Zange von dorsolateral, c nach Entfernung, $d F L$, vFL dorsale und ventrale Facies lunata, FA Fossa acetabuli, FK Femurkopf, LCF Lig. capitis femoris, $\mathrm{OCH}$ Osteochondrom. (Mit Genehmigung von M. Dienst)

dorsal. Entsprechende MR-Tomogramme sind daher insbesondere auf direkte oder indirekte Zeichen einer solchen Läsion hin zu analysieren. Im MR-Arthrogramm sieht man häufig recht gut die beginnende Dissoziation des Labrum-Knorpel-Übergangs, während man im konventionellen MR meist nur die Labrumdegeneration, Labrumzysten und Azetabulumerkerzysten erkennt [47].

Vor der Indikationsstellung zur arthroskopischen Behandlung ist die zugrunde liegende Ursache zu identifizieren, da sie in der Regel das operative Verfahren festlegt:

- Bei isolierten traumatischen Rupturen würden wir zumindest primär arthroskopisch vorgehen.

- Beim femoroazetabulären Impingement hängt die Entscheidung von der Lokalisation und dem Schweregrad der Veränderung an Pfanne oder Femurkopf ab (s. unten).

- Bei Vorliegen einer Hüftdysplasie wird das Gelenk in der Regel zunächst arthroskopiert, um dann die Entscheidung für ein zweizeitiges, umstellendes Operationsverfahren zu treffen.

Ähnlich der Meniskuschirurgie am Kniegelenk gilt der weitestgehende Erhalt des Labrum acetabulare als primäres Ziel. In verschiedenen biomechanischen Studien konnte nachgewiesen werden, dass es eine wesentliche Rolle in der Hüftstabilität spielt $[25,54]$. Es wurde zudem gezeigt, dass das es für eine homogene Gelenkflüssigkeitsverteilung wichtig ist [25].
Aus diesen Gründen erscheint es wichtig, das Labrum nur bei ausgedehnten Rissen und schwerer Degeneration zu resezieren. Sowohl bei arthroskopischem als auch offenem Vorgehen entfernen wir daher in der Regel nur seine instabilen Anteile bzw. die des angrenzenden Knorpels. Sollte dieser bis auf den darunter liegenden subchondralen Knochen entfernt werden, wird arthroskopisch oder offen mikrofrakturiert. Bei der Hüftdysplasie finden sich häufig instabile Längsrisse oder invertierte Labrumanteile. Auch hier sollte das Labrum nur bis auf einen weitestgehend stabilen Rand zurückgetrimmt werden, um die „letzte Leitplanke“ der Dysplasie nicht zu entfernen [46]. In der meist anstehenden sekundären Umstellungsoperation wird das Labrum mit dem angrenzenden Knorpel aus der direkten Belastungszone herausgedreht.

Instabile Labrumanteile können in arthroskopischer Technik mit Nahtankern refixiert werden [35], die Notwendigkeit zur Refixation des Labrums ohne vorherige Ablösung durch den Operateur stellt unserer Erfahrung nach jedoch eine Seltenheit dar. Häufiger ist sie erforderlich, wenn das Labrum abgelöst wurde, um den knöchernen Pfannenrand zurückzutrimmen.

Ähnlich dem Kniegelenkmeniskus ist die Basis des Labrum acetabulare gut genug durchblutet, um ein Anheilen zu ermöglichen [40]. Das arthroskopische Débridement von instabilen Labrum- und Knorpelanteilen führen wir unter Traktion durch. Nur so können die in der Regel auf der zentralen Seite liegenden Lappen mit abgewinkelten Shavern und Zangen bzw. Küretten entfernt werden.

Die Ergebnisse sowohl der arthroskopischen als auch der offenen Labrumchirurgie hängen primär vom Zustand des Gelenkknorpels ab. Die Behandlung traumatischer Läsionen des Labrum-KnorpelÜbergangs führt häufig zu guten Ergebnissen. Bei ausgedehnten Knorpelschäden und degenerativen Gelenkprozessen sind die Resultate weniger gut ( $\mathbf{0}$ Abb. 4) [44].

\section{Läsionen des hyalinen Gelenkknorpels}

Isolierte traumatische Knorpelläsionen am Hüftgelenk sind selten. Häufig sind sie mit anderen Verletzungen assoziiert, wie mit Rupturen des Lig. capitis femoris oder Labrumrupturen nach Subluxationen oder Luxationen, oder sie kommen nach direkten seitlichen Anpralltraumen vor [7]. Unserer Erfahrung nach sind dies vorteilhafte Indikationen zur arthroskopischen Intervention. Die instabilen Knorpelareale können entfernt, assoziierte Rupturen des Lig. capitis femoris oder anderer Strukturen reseziert und der darunter liegende Knochen mikrofrakturiert werden [11, 18].

Eigene Erfahrungen mit der autologen Knorpelzelltransplantation am Hüftgelenk haben wir bislang nicht. Derartige Verfahren befinden sich momentan sicherlich noch im experimentellen Stadium und sind nicht etabliert. Die Indikationen zur Knorpelzelltransplantation an der Hüfte sind selten, hier wäre zur- 
Abb. 3 Typische Läsion des ventrosuperioren Knorpel-Labrum-Übergangs beim a femoroazetabulären Impingement, b Lappenresektion und Abrasionsplastik mit dem Shaver, FK Femurkopf, FL Facies lunata, $K$ frei liegender subchondraler Knochen, KL Knorpellappen, vs $L$ ventrosuperiores Labrum. (Mit Genehmigung von M. Dienst)
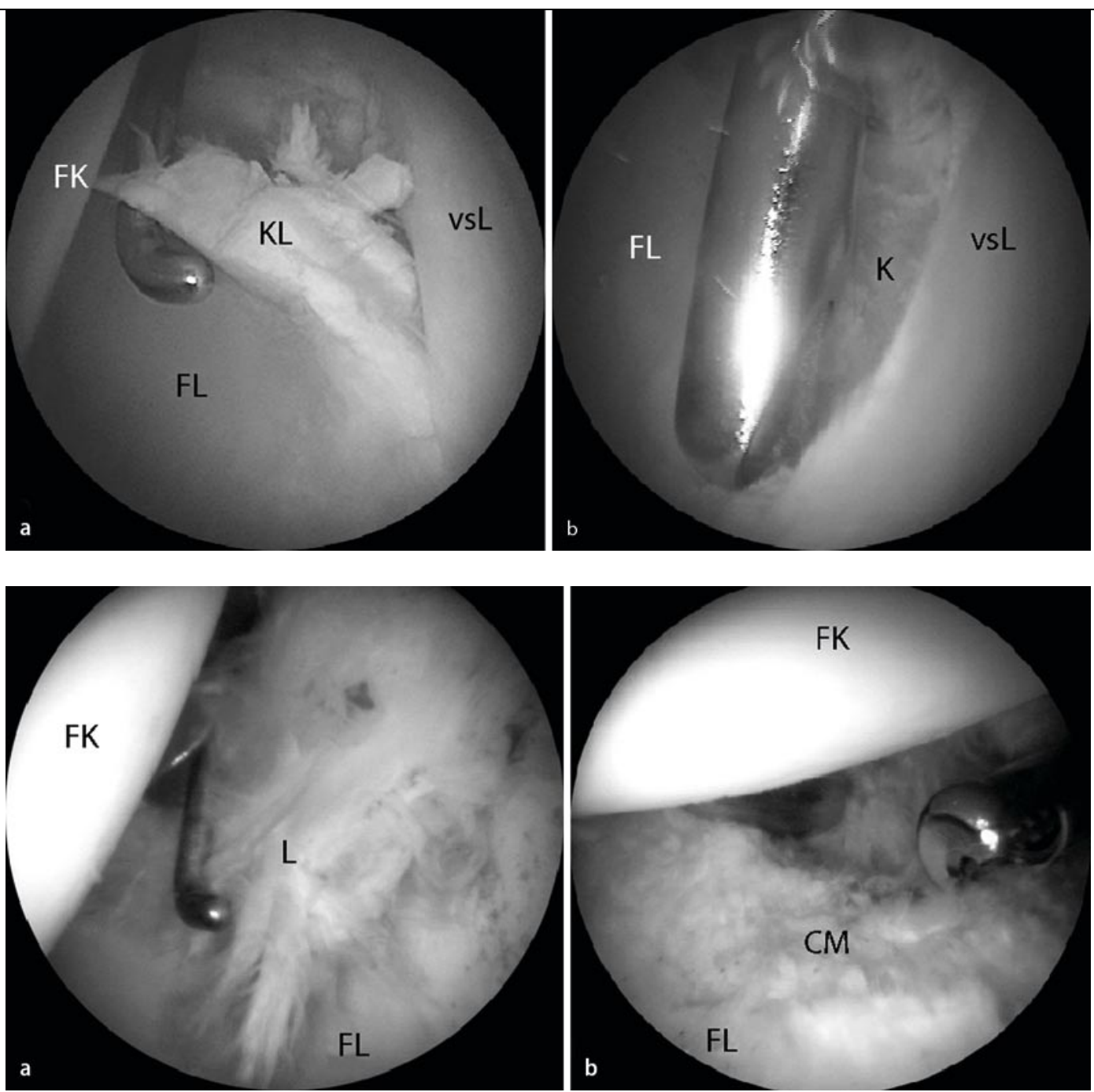

Abb. 4 Degenerativer superiorer Labrumkomplexriss vor (a) und nach Resektion (b), CM Chondromalazie, FK Femurkopf, FL Facies lunata, $L$ Labrum. (Mit Genehmigung von $M$. Dienst)

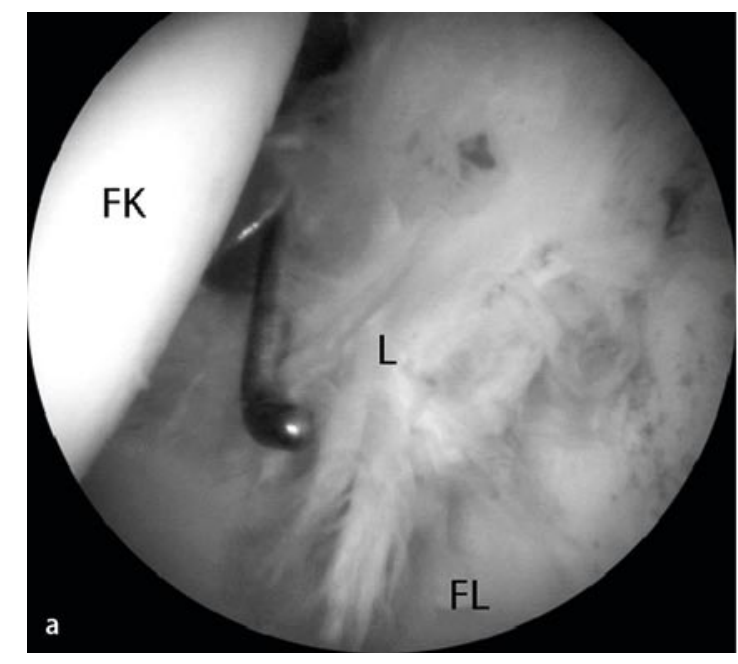

Alter und röntgenologischen Befund individuell zu treffen.

Bei initialen oder moderaten degenerativen Veränderungen gibt es häufig Indikationen zum arthroskopischen Vorgehen. Nicht selten finden sich assoziierte pathologische Veränderungen wie freie osteochondrale Fragmente, degenerative Rupturen des Lig. capitis femoris [32], Läsionen des Labrum acetabulare, reaktiventzündliche Veränderungen der Gelenkschleimhaut und mechanisch störende Osteophyten [18]. Wie in anderen Gelenken kann ein arthroskopisches Débridement mit entsprechender Behandlung der assoziierten Läsion zur deutlichen und anhaltenden Besserung der Beschwerden führen $[18,48]$.

Bei degenerativen Veränderungen und gleichzeitiger deutlicher Deformität wie bei einem ausgeprägten femoroazetabulären Impingement oder nach einen Morbus Perthes oder einer Epiphyseolysis ca- pitis femoris kann dagegen auch ein offenes Vorgehen empfehlenswert sein, um die mechanische Situation des Gelenks effizient zu verbessern. In diesen Fällen sind die Möglichkeiten des arthroskopischen Rekonturierens eingeschränkt. Gelegentlich erlaubt es die Arthroskopie, den Schweregrad und die Ausdehnung der degenerativen Veränderungen zu beurteilen. Nicht selten findet sich dann ein schlechterer Zustand des Gelenkknorpels, als präoperativ zu vermuten gewesen wäre. In solchen Situationen kann die Arthroskopie helfen, die Entscheidung für einen früheren endoprothetischen Ersatz zu stellen.

\section{Ruptur des Lig. capitis femoris}

Die genaue Funktion dieses Ligaments bleibt unbekannt. Während die zentrale Arterie beim Kleinkind für die Vaskularisation der Femurepiphyse noch wichtig 


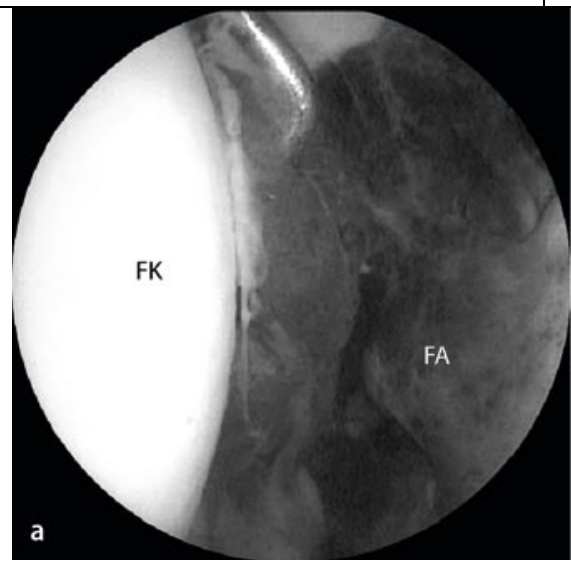

Indikationen zur Arthroskopie großer Gelenke

Abb. $5 \Delta$ A Fossa acetabuli, a Synovitis, b Synovektomie mit flexibler Thermoelektrode, c nach Synovektomie, FA Fossa acetabuli, FK Femurkopf, FL Facies lunata. (Mit Genehmigung von M. Dienst)

erscheint, wurde in anatomischen Studien nachgewiesen, dass sie beim Erwachsenen keine Rolle mehr spielt [27]. Immunhistochemische Studien zeigten eine hohe Anzahl freier Nervenendigungen, welche auf eine vorwiegend propriozeptive Funktion dieses Ligaments hinweisen [39].

Rupturen des Lig. capitis femoris spielen unserer Erfahrung nach eine nicht unerhebliche klinische Rolle. Es ist dabei unbedingt zu berücksichtigen, dass sie sowohl traumatischer als auch nichttraumatischer Genese sein können [12, 32]. Bei Hüftluxationen kommt es wahrscheinlich ausnahmslos zu einer Komplett- oder zumindest Teilruptur des Lig. capitis femoris. Rotationsverletzungen mit möglichen Subluxationen führen anscheinend recht häufig zu Distorsionen, Partial- und sogar Komplettrupturen des Ligaments. Hier steht vermutlich das Außenrotationstrauma als Ursache im Vordergrund. Auch chronische Mikrotraumen müssen als möglicher Grund bedacht werden [38]. Bei den nichttraumatischen Rupturen stehen Hüftdysplasien und Kopfdeformitäten nach einem Morbus Perthes an erster Stelle (ब Abb. 1). Insbesondere bei der Hüftdysplasie sollte daher unserer Erfahrung nach vor einer Umstellungsosteotomie eine Arthroskopie erfolgen, um einem schlechten Ergebnis durch ein mechanisch irritierendes rupturiertes Lig. capitis femoris vorzubeugen.

Vor allem auch im Hinblick auf die präoperativ oft unklare Diagnose ist der Verdacht auf eine Ruptur des Lig. capitis femoris eine klare Indikation für die arthroskopische Intervention. Arthroskopisch muss das Ligament unter Innen- und Außenrotation inspiziert werden. Bei Außenrotation spannt es sich an, bei Innenrotation erschlafft es. Die vollständige arthroskopische Entfernung gelingt über das Einbringen von abgewinkelten Zangen und Shavern über das ventrale und dorsolaterale Portal. Die Ergebnisse der arthroskopischen Resektion sind bei guten Knorpelverhältnissen exzellent [6, 32].

\section{Synoviale Erkrankungen}

Differenzialdiagnostisch müssen neben reaktiven Synovitiden berücksichtigt werden:

- Erkrankungen des rheumatischen Formenkreises,

- eine synoviale Chondromatose und

- eine pigmentierte villonoduläre Synovitis (PVNS).

Die Arthroskopie ist ein ausgezeichnetes Verfahren zur genauen Inspektion der Gelenkschleimhaut und zur Biopsie veränderter Areale. Das bei der Anlage des Portals gewonnene Punktat kann zur weiteren Analyse auf Kristalle oder Zellen verwendet werden. Darüber hinaus hat sich die arthroskopische Technik so weit entwickelt, dass große Teile der Synovialmembran arthroskopisch entfernt werden können. Die Fossa acetabuli kann fast vollständig synovektomiert werden, mit von außen steuerbaren Hochfrequenzelektroden kann das Pulvinar bis hin zum Lig. transversum entfernt werden (• Abb. 5). Wichtig ist auch hier die Kombination des ventralen und dorsolateralen Portals. In der Gelenkperipherie können die ventralen Areale einfach erreicht werden, die dorsale Kammer ist dagegen schwer zugänglich.

Auch bei Vorliegen einer synovialen Chondromatose oder PVNS empfiehlt sich ein arthroskopisches Vorgehen. Unter Verwendung von dickeren Portalhülsen können auch größere Chondrome entfernt werden, notfalls müssen sie zerkleinert werden ( $\boldsymbol{\bullet}$ Abb. 2). Im Fall einer PVNS kann präoperativ keine sichere Diagnose gestellt werden. Die Arthroskopie dient dann zur Biopsie und Diagnosesicherung, gleichzeitig führen wir bei der lokalen PVNS eine Entfernung des Herdes durch.

\section{Femoroazetabuläres Impingement (FAI)}

Die Einführung des FAI-Konzepts durch Ganz et al. [26] hat die Hüftchirurgie in den vergangenen Jahren zunehmend geprägt. Bis vor wenigen Jahren wurden Veränderungen auf der azetabulären (Pincer- oder Beißzangen-FAI) und femoralen Seite (Cam- oder Nockenwellen-FAI) ausschließlich durch die von Ganz et al. [26] propagierte digastrische Trochanterosteotomie angegangen [33]. Trotz des eleganten und anatomisch respektvollen $\mathrm{Zu}$ gangs und der hervorragenden Übersicht und Erreichbarkeit des gesamten Hüftgelenks haben sich mit Hinblick auf die Invasivität des Verfahrens und die langwierige Rehabilitation arthroskopische Techniken zur Behandlung des FAI entwickelt.

Wie bereits oben beschrieben konkurrieren in unserer Klinik die arthrosko- 
Abb. 6 Femoroazetabuläres Impingement mit Kopf-Hals-Offsetverlust ventrolateral vor $\mathbf{a}$ und $\mathbf{b}$ nach Rekontuierung, B Bump, FKK Femurkopfknorpel, I lateral, $M K, V K$ mediale und ventrale Kapsel, SH Schenkelhals, $v$ ventral, $v L, V L$ ventrales Labrum. (Mit Genehmigung von M. Dienst)

Abb. 7 Psoassehne vor (a) und nach Tenotomie (b) via peripherem Gelenkkompartiment, $E$ Elektrode, $K$ Kapsel, MI M. iliacus, $P$ Psoassehne. (Mit Genehmigung von M. Dienst)
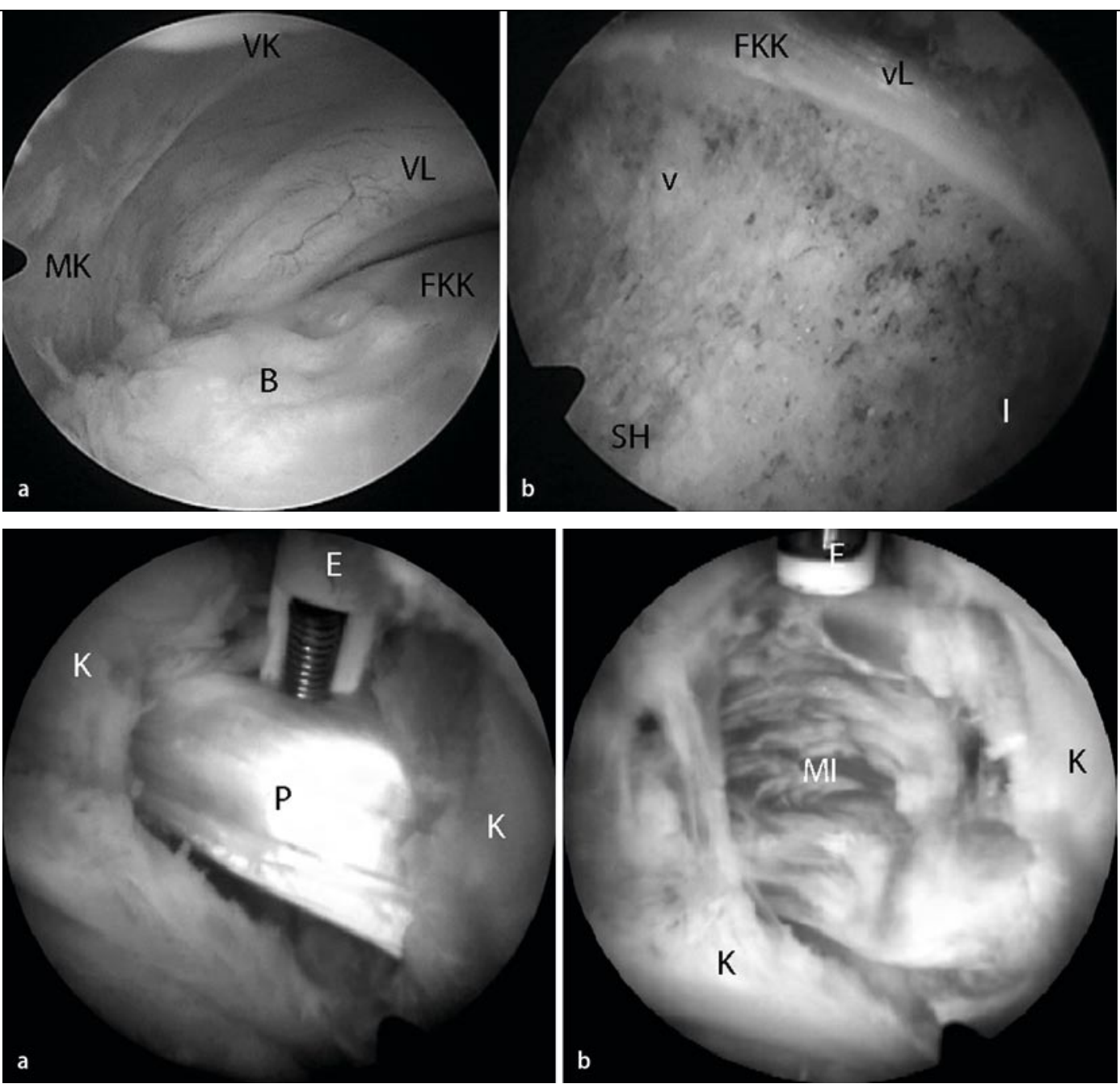

pischen und die offenen Verfahren nur in Grenzbereichen. Wenngleich wir davon überzeugt sind, dass die Arthroskopie das offene Vorgehen zunehmend zurückdrängen wird, gibt es unserer Erfahrung nach klare Indikationen jeweils für ein arthroskopisches oder offenes Vorgehen: Grundsätzlich gehen wir bei Vorliegen eines deutlichen Pincer-FAI eher offen vor. Eine korrekte Ablösung des Labrum acetabulare mit konsekutivem $\mathrm{Zu}$ rücktrimmen des Pfannenrandes und Refixieren des Labrums mit Ankern ist zum gegenwärtigen Zeitpunkt nur offen sicher durchführbar. Wenngleich erste Versuche zur Refixation eines instabilen Labrumanteils mit Nahtankern gelangen [35], sind weitere technische Verbesserungen und mehr arthroskopische Erfahrung notwendig, um das Labrum über einen größeren Bereich abzulösen und wieder sicher anzuheften. Anders verhält es sich bei einem verkalkten Labrum acetabulare, dieses kann zusammen mit dem
Pfannenrand arthroskopisch zurückgefräst werden. Bei einem nur gering ausgeprägten Pincer-FAI mit geringer Pfannenretroversion ist individuell zu besprechen, ob nicht eine arthroskopische Offsetverbesserung auf der femoralen Seite ausreichen würde.

Bei Vorliegen eines Cam-FAI gehen wir dagegen eher arthroskopisch vor, insbesondere wenn der Kopf-Hals-Offsetverlust ventral und ventrolateral liegt (- Abb.6).

Bei weit lateral und dorsolateral liegendem Bump und einer schweren Deformität wie nach einer Epiphyseolysis capitis femoris oder ggf. einem Morbus Perthes bevorzugen wir zum gegenwärtigen Zeitpunkt noch das offene Vorgehen, hier können die Gefäßeintrittsstellen sicherer dargestellt und größere Kopfanteile in kürzerer Zeit zurückgetrimmt werden.

\section{Psoassehnentendinitis und schmerzhaftes Springen der Psoassehne}

In den seltenen Fällen einer persistierenden Psoastendinitis oder schmerzhaft springenden Psoassehne trotz physiotherapeutischer Therapie kann eine Tenotomie indiziert sein. Offene Verfahren zeigten Komplikationsraten bis zu $40 \%$ wie Rezidive, eine bleibende Schwäche der Hüftbeugung und Parästhesien im ventralen Oberschenkel $[1,23,34,45]$. Byrd [8, 9] berichtete kürzlich von einer extraartikulär endoskopischen Tenotomie am Trochanter minor mit guten klinischen Resultaten. Wir führen diese Technik nach endoprothetischer Versorgung mit entsprechenden Psoasproblemen durch, bevorzugen in den meisten anderen Fällen aber eine Tenotomie ausgehend von der Gelenkperipherie [52]. Hier lässt sich die Psoassehne leicht durch eine kleine Inzision der 
ventromedialen Kapsel identifizieren und selektiv durchtrennen (• Abb. 7).

\section{Seltene Indikationen}

Nur selten stellen sich Indikationen zur arthroskopischen Intervention bei Vorliegen einer idiopathischen Hüftkopfnekrose, beim Morbus Perthes oder Komplikationen des endoprothetischen Gelenkersatzes. Bei der Hüftkopfnekrose oder dem Morbus Perthes können assoziierte Läsionen wie ein freier Körper arthroskopisch angegangen werden. Eine Kontrolle der Kopfdekompression führen wir navigiert oder fluoroskopisch kontrolliert und nicht unter arthroskopischer Kontrolle durch. Die Osteochondrosis dissecans am Hüftgelenk ist selten, bei Vorliegen je nach Stadium aber gut arthroskopisch zu debridieren oder anzubohren.

Die insbesondere im Kindesalter auftretende septische Arthritis stellt eine weitere seltene, aber gute Indikation für eine arthroskopische Spülung und Débridement dar [3, 4, 14]. Der Keim kann isoliert und entsprechend antibiotisch abgedeckt werden, das Gelenk wird zentral und peripher gespült und débridiert, in das periphere Kompartiment kann eine Dränage eingelegt werden.

\section{Kontraindikationen}

Risikoreich erscheint die Arthroskopie bei frischer Azetabulumfraktur. Hier berichteten Bartlett et al. [2] von einer Flüssigkeitsextravasation ins Retroperitoneum mit Herzstillstand. Wir würden in einem solchen Fall einige Wochen abwarten und mit möglichst niedrigem Druck (ggf. nur Schwerkraftspülung) arbeiten. Nicht sinnvoll ist ein arthroskopisches Vorgehen bei bereits fortgeschrittenen degenerativen Veränderungen, insbesondere wenn diese bereits mit einem schlechten Bewegungsumfang, v. a. einer Rotationskontraktur, kombiniert sind. Der Sinn eines arthroskopischen oder offenen Débridements ist hier in Frage zu stellen. Zudem ist zu berücksichtigen, dass solche Gelenke meist nicht mehr ausreichend distrahiert werden können.

Bei einer deutlichen Bewegungseinschränkung ohne degenerative Veränderungen ist meist ein offenes Verfahren zu bevorzugen. Die Kapsel kann dabei labrumnah zirkumferent inzidiert werden, gleichzeitig können in kürzerer Operationszeit auch andere Probleme angegangen werden.

Bei einer Coxa profunda und einem fortgeschrittenen Pincer-Impingement ist der Zugang zum zentralen Kompartiment nicht selten erschwert. Auch hier sollte ein offenes Verfahren in Betracht gezogen werden. Bei einer gleichzeitigen Labrumverkalkung kann die Arthroskopie ausschließlich ohne Traktion durchgeführt werden. Ausgehend von der Gelenkperipherie können das Kopf-HalsOffset verbessert und das verkalkte Labrum mit Pfannenrandanteilen zurückgetrimmt werden. Bei adipösen Patienten muss überprüft werden, ob ggf. überlange Arthroskope vorhanden sind, um bis ins Gelenk vorzudringen.

\section{Korrespondenzadresse}

\section{Dr. M. Kusma}

Klinik für Orthopädie

und Orthopädische Chirurgie,

Universitätskliniken des Saarlandes,

66421 Homburg, Saar

mkusma@gmx.de

Interessenkonflikt. Der korrespondierende Autor gibt an, dass kein Interessenkonflikt besteht.

\section{Literatur}

1. Allen WC, Cope R (1995) Coxa saltans: the snapping hip revisited. J Am Acad Orthop Surg 3: 303308

2. Bartlett CS, DiFelice GS, Buly R et al. (1998) Cardiac arrest as a result of intraabdomial extravasation of fluid during arthroscopic removal of a loose body from the hip joint of a patient with an acetabular fracture. J Orthop Trauma 12: 294-300

3. Blitzer CM (1993) Arthroscopic management of septic arthritis of the hip. Arthroscopy 9: 414-416

4. Bould M, Edwards D,Villar RN (1993) Arthroscopic diagnosis and treatment of septic arthritis of the hip joint. Case report. Arthroscopy 9: 707-708

5. Byrd JWT (1994) Hip arthroscopy utilizing the supine position. Arthroscopy 10: 275-280

6. Byrd JWT (1998) Arthroscopy of select hip lesions. In: Byrd JWT (ed) Operative hip arthroscopy. Thieme, Stuttgart New York, pp 153-171

7. Byrd JWT (2001) Lateral impact injury: a source of occult hip pathology. Clin Sports Med 20: 801-816

8. Byrd JWT (2004) Hip arthroscopy: evolving frontiers. Operat Tech Orthop 14: 58-67

9. Byrd JWT (2005) Snapping hip. Operat Tech Sports Med 13: 46-54

10. Byrd JWT, Chern KY (1997) Traction versus distension for distraction of the joint during hip arthroscopy. Arthroscopy 13: 346-349
11. Byrd JWT, Jones KS (2002) Osteoarthritis caused by an inverted acetabular labrum: radiographic diagnosis and arthroscopic treatment. Arthroscopy 18: 741-747

12. Byrd JWT, Jones KS (2004) Traumatic rupture of the ligamentum teres as a source of hip pain. Arthroscopy 20: 385-391

13. Byrd JWT, Pappas JN, Pedley MJ (1995) Hip arthroscopy: an anatomic study of portal placement and relationship to the extra-articular structures. Arthroscopy 11: 418-423

14. Chung WK, Slater GL, Bates EH (1993) Treatment of septic arthritis of the hip by arthroscopic lavage. J Pediatr Orthop 13: 444-446

15. Dienst M (2005) Hip arthroscopy: technique and anatomy. Operat Tech Sports Med 13: 13-23

16. Dienst M, Kohn D (2001) Hüftarthroskopie. Minimal-invasive Diagnostik und Therapie des erkrankten und verletzten Hüftgelenks. Unfallchirurg 104: 2-18

17. Dienst M, Kohn D (2003) Arthroskopie. In: Wirth CJ, Zichner L (Hrsg) Orthopädie und Orthopädische Chirurgie. In: Tschauner C (Hrsg) Becken, Hüfte. Thieme, Stuttgart New York, S 78-81

18. Dienst M, Seil R, Gödde S et al. (1999) Hüftarthroskopie bei radiologisch beginnender bis mäßiggradiger Koxarthrose. Diagnostische und therapeutische Wertigkeit. Orthopäde 28: 812-818

19. Dienst M, Gödde S, Seil R et al. (2001) Hip arthroscopy without traction: in vivo anatomy of the peripheral hip joint cavity. Arthroscopy 17: 924-931

20. Dienst M, Gödde S, Seil R et al. (2002) Diagnostische Arthroskopie des Hüftgelenks. Operat Orthop Traumatol 14: 1-15

21. Dienst M, Seil R, Gödde S et al. (2002) Effects of traction, distension, and joint position on distraction of the hip joint: an experimental study in cadavers. Arthroscopy 18: 865-871

22. Dienst M, Seil R, Kohn D (2005) Safe arthroscopic access to the central compartment of the hip joint Arthroscopy 21: 1510-1514

23. Dobbs MB, Gordon JE, Luhmann SJ et al. (2002) Surgical correction of the snapping iliopsoas tendon in adolescents. J Bone Joint Surg Am 84-A: 420-424

24. Dorfmann H, Boyer T (1999) Arthroscopy of the hip: 12 years of experience. Arthroscopy 15: 67-72

25. Ferguson SJ, Bryant JT, Ganz R et al. (2003) An in vitro investigation of the acetabular labral seal in hip joint mechanics. J Biomech 36: 171-178

26. Ganz R, Parvizi J, Beck M et al. (2003) Femoroacetabular impingement: a cause for osteoarthritis of the hip. Clin Orthop Relat Res 417: 112-120

27. Gautier E, Ganz K, Krügel N et al. (2000) Anatomy of the medial femoral circumflex artery and its surgical implications. J Bone Joint Surg Br 82-B: 679683

28. Glick JM, Sampson TG, Behr JT et al. (1987) Hip arthroscopy by the lateral approach. Arthroscopy 3 : 4-12

29. Goldman A, Minkoff J, Price A et al. (1987) A posterior arthroscopic approach to bullet extraction from the hip. J Trauma 27: 1294-1300

30. Gondolph-Zink B (1992) Aktueller Stand der diagnostischen und operativen Hüftarthroskopie. Orthopäde 21: 249-256

31. Gondolph-Zink B, Degenhart M (1998) Arthroskopische Behandlung der Osteochondrosis dissecans am Hüftgelenk. Arthroskopie 11: 200-202

32. Gray AJR, Villar RN (1997) The ligamentum teres of the hip: an arthroscopic classification of its pathology. Arthroscopy 13: 575-578

33. Gross RH (1977) Arthroscopy in hip disorders in children. Orthop Rev 6: 43-49 
34. Hoskins JS, Burd TA, Allen WC (2004) Surgical correction of internal coxa saltans. A 20-year consecutive study. Am J Sports Med 32: 998-1001

35. Kelly BT, Williams RJ 3rd, Philippon MJ (2003) Hip arthroscopy: current indications, treatment options, and management issues. Am J Sports Med 31: 1020-1037

36. Klapper R, Dorfmann H, Boyer T (1998) Hip arthroscopy without traction. In: Byrd JWT (ed) Operative hip arthroscopy. Thieme, Stuttgart New York, pp 139-152

37. Kuklo TR,Mackenzie WG, Keeler KA (1999) Hip arthroscopy in Legg-Calve-Perthes disease. Case report. Arthroscopy 15: 88-92

38. Kusma M, Jung J, Dienst M et al. (2004) Arthroscopic treatment of an avulsion fracture of the ligamentum teres of the hip in an 18-year-old horse rider. Arthroscopy [Suppl 2] 20: 64-66

39. Leunig M, Beck M, Stauffer E et al. (2000) Free nerve endings in the ligamentum capitis femoris. Acta Orthop Scand 71: 452-454

40. McCarthy J, Noble P, Aluisio FV et al. (2003) Anatomy, pathologic features, and treatment of acetabular labral tears. Clin Orthop Relat Res 406: 38-47

41. Okada Y, Awaya G, Ikeda T et al. (1989) Arthroscopic surgery for synovial chondromatosis of the hip. J Bone Joint Surg Br 71: 198-199

42. Rittmeister M, Hochmuth K, Kriener S et al. (2005) 5-Jahres-Ergebnisse nach autologer Knorpel-Knochen-Transplantation bei Hüftkopfnekrose. Orthopäde 34: 320-326

43. Sampson TG, Farjo LA (1998) Hip arthroscopy by the lateral approach: technique and selected cases. In: Byrd JWT (ed) Operative hip arthroscopy. Thieme, Stuttgart New York, pp 105-122

44. Santori N, Villar RN (2000) Acetabular labral tears: result of arthroscopic partial limbectomy. Arthroscopy 16: 11-15

45. Schaberg JE, Harper MC, Allen WC (1984) The snapping hip syndrome. Am J Sports Med 12: 361-365

46. Tschauner C, Hofmann S (1998) Labrumläsion bei der Restdysplasie des Hüftgelenks. Biomechanische Überlegungen zur Pathogenese und Behandlung. Orthopäde 27: 725-732

47. Urban M, Hofmann S, Tschauner C et al. (1998) MR-Arthrographie bei der Labrumläsion des Hüftgelenks. Technik und Stellenwert. Orthopäde 27: 691-698

48. Villar RN (1991) Arthroscopic debridement of the hip: a minimally invasive approach to osteoarthritis. J Bone Joint Surg Br 73: 170-171

49. Villar RN (1992) Hip arthroscopy. Butterworth Heinemann, Oxford

50. Villar RN (1992) Hip arthroscopy. Br J Hosp Med 47: 763-766

51. Villar RN (1994) Arthroscopy. BMJ 308: 51-53

52. Wettstein M, Jung J, Dienst M (2006) Arthroscopic psoas tenotomy. Arthroscopy 22: 907

53. Wettstein M, Gödde S, Morgenthaler K et al. (in press) Distraction of the hip joint utilizing the supine and lateral position. Arthroscopy in press

54. Wingstrand H, Wingstrand A, Krantz P (1990) Intracapsular and atmospheric pressure in the dynamics and stability of the hip. A biomechanical study. Acta Orthop Scand 61: 231-235

55. Witwity T, Uhlmann RD, Fischer J (1988) Arthroscopic management of chondromatosis of the hip joint. Arthroscopy 4: 55-56 